\title{
ON THE ESTIMATION OF THE $L_{2}$-NORM OF A FUNCTION OVER A BOUNDED SUBSET OF $\boldsymbol{R}^{n}$
}

\author{
HOMER F. WALKER
}

\begin{abstract}
The objective of this paper is to present an estimate bounding the $L_{2}$-norm of a function over a bounded subset of $\boldsymbol{R}^{n}$ by the $L_{2}$-norms of its derivatives of arbitrary order over all of $\boldsymbol{R}^{n}$ and the $L_{2}$-norm of its projection onto a finite-dimensional space of functions with bounded support. The estimate essentially generalizes inequalities of Friedrichs [1, p. 284] and Lax and Phillips [2, p. 95]. An application of the estimate is made to the Fredholm theory of elliptic partial differential operators in $\boldsymbol{R}^{n}$.
\end{abstract}

1. Introduction. Let $L_{2}\left(\boldsymbol{R}^{n} ; \boldsymbol{C}^{k}\right)$ be the usual Hilbert space of equivalence classes of $\boldsymbol{C}^{k}$-valued functions on $\boldsymbol{R}^{n}$ whose absolute values are Lebesguesquare-integrable over $\boldsymbol{R}^{n}$. Denote the norm on $L_{2}\left(\boldsymbol{R}^{n} ; \boldsymbol{C}^{k}\right)$ by \|\| . For each positive integer $m$, let $H_{m}\left(\boldsymbol{R}^{n} ; \boldsymbol{C}^{k}\right)$ be the Hilbert space consisting of those elements of $L_{2}\left(R^{n} ; C^{k}\right)$ which have strong partial derivatives of order $m$ in $L_{2}\left(\boldsymbol{R}^{n} ; \boldsymbol{C}^{k}\right)$. To specify the norm on $H_{m}\left(\boldsymbol{R}^{n} ; \boldsymbol{C}^{k}\right)$, first define

$$
\left\|\partial_{x}^{m} u\right\|=\left\{\int_{n}|\xi|^{2 m}|\hat{u}(\xi)|^{2} d \xi\right\}^{1 / 2}
$$

for an element $u$ of $H_{m}\left(\boldsymbol{R}^{n} ; \boldsymbol{C}^{k}\right)$, where

$$
\hat{u}(\xi)=(2 \pi)^{-n / 2} \int_{R^{n}} e^{-i \xi x} u(x) d x
$$

is the Fourier transform of $u$. Then take the norm of an element $u$ of $H_{m}\left(\boldsymbol{R}^{n} ; \boldsymbol{C}^{k}\right)$ to be $\|u\|_{m}=\left\{\|u\|^{2}+\left\|\partial_{x}^{m} u\right\|^{2}\right\}^{1 / 2}$. Given a subset $D$ of $\boldsymbol{R}^{n}$, denote by $L_{2}\left(D ; C^{k}\right)$ and $H_{m}\left(D ; C^{k}\right)$ the subspaces of $L_{2}\left(R^{n} ; C^{k}\right)$ and $H_{m}\left(\boldsymbol{R}^{n} ; \boldsymbol{C}^{k}\right)$, respectively, consisting of elements with support in $D$.

Consider the following theorems.

TheOREM (Friedrichs [1, p. 284]). If a bounded subset $D$ of $\boldsymbol{R}^{n}$ and $a$ number $\varepsilon>0$ are given, then there exist an integer $M>0$ and elements

Received by the editors November 1,1971 .

AMS (MOS) subject classifications (1969). Primary 35B45, 35J45, 35F05.

Key words and phrases. Calculus inequalities, Friedrichs inequality, first-order elliptic operators, indices of elliptic operators, Fredholm operators.

(C) American Mathematical Society 1973 
$w_{1}, \cdots, w_{M}$ of $L_{2}\left(D ; C^{k}\right)$ such that the estimate

holds for all $u$ in $H_{1}\left(D ; C^{k}\right)$.

$$
\|u\|^{2} \leqq \varepsilon\left\|\partial_{x}^{1} u\right\|^{2}+\sum_{j=1}^{M}\left|\left(u, w_{j}\right)\right|^{2}
$$

Theorem (LAX, Phillips [2, p. 95]). If a number $R>0$ is given and if $n \geqq 3$, then the estimate

$$
\int_{|x| \leqq R}|u(x)|^{2} d x \leqq \frac{R^{2}}{2(n-2)}\left\|\partial_{x}^{1} u\right\|^{2}
$$

holds for all $u$ in $H_{1}\left(\boldsymbol{R}^{n} ; \boldsymbol{C}^{k}\right)$.

The first objective of the following is to demonstrate a general estimate which, despite a certain lack of sharpness, includes in essence the two estimates above as special cases. The second objective is to apply this estimate toward obtaining new bounds on the Fredholm index of a linear elliptic first-order partial differential operator in $\boldsymbol{R}^{2}$ whose coefficients become constant outside a bounded subset of $\boldsymbol{R}^{2}$.

2. The estimate. For each positive integer $p$, denote by $\pi_{p}$ the projection onto the subspace of $L_{2}\left(R^{n} ; C^{k}\right)$ spanned by functions of the form $x^{\alpha} X_{R}^{n}(x) c$, where $\alpha$ is a multi-index with $0 \leqq|\alpha|<p, c$ is a vector in $C^{k}$, and $X_{R}^{n}$ is the characteristic function of the ball $B_{R}^{n}=\left\{x \in \boldsymbol{R}^{n}:|x| \leqq R\right\}$, i.e.,

$$
\begin{aligned}
X_{R}^{n}(x) & =1 & & \text { if }|x| \leqq R, \\
& =0 & & \text { if }|x|>R .
\end{aligned}
$$

Denote by $\pi_{0}$ the zero operator on $L_{2}\left(R^{n} ; C^{k}\right)$. Then one has the following estimate.

THEOREM. Suppose that there are given positive integers $n$ and $m$ and $a$ number $\varepsilon, 0<\varepsilon<1$. Then there exists a calculable constant $C_{n, m, \varepsilon}$ depending on $n, m$, and $\varepsilon$ for which the estimate

$$
\int_{|x| \leqq R}|u(x)|^{2} d x \leqq\left\|\pi_{p} u\right\|^{2}+\frac{C_{n, m, \varepsilon} R^{2 m}}{(2 p+n-2 m)^{2 m(1-\varepsilon)}}\left\|\partial_{x}^{m} u\right\|^{2}
$$

holds for all nonnegative integers $p>(m-n / 2)$ and all $u$ in $H_{m}\left(\boldsymbol{R}^{n} ; \boldsymbol{C}^{k}\right)$.

Before proving the theorem, the lemma below will be established. It will be seen that the theorem, the proof of which follows the proof of the lemma, is an easy corollary to the lemma.

Lemma. Suppose that there are given positive integers $n$ and $m$ and $a$ number $\varepsilon, 0<\varepsilon<1$. Then there exists a calculable constant $C_{n, m, \varepsilon}$ depending 
on $n, m$, and $\varepsilon$ for which the estimate

$$
\int_{R^{n}}|\xi|^{-2 m}|\hat{\phi}(\xi)|^{2} d \xi \leqq \frac{C_{n, m, \varepsilon} R^{2 m}}{(2 p+n-2 m)^{2 m(1-\varepsilon)}}\|\phi\|^{2}
$$

holds for all $\phi$ in $\left(I-\pi_{p}\right)\left(L_{2}\left(B_{R}^{n} ; C^{k}\right)\right)$ and all nonnegative integers $p>(m-n / 2)$.

Proof of The Lemma. Let $p$ be a nonnegative integer greater than $(m-n / 2)$ and let $\phi$ be an element of $\left(I-\pi_{p}\right)\left(L_{2}\left(B_{R}^{n} ; C^{k}\right)\right)$. Then for any $K>0$,

$$
\begin{aligned}
\int_{R^{n}}|\xi|^{-2 m}|\hat{\phi}(\xi)|^{2} d \xi & \\
& =\int_{|\xi| \geqq K}|\xi|^{-2 m}|\hat{\phi}(\xi)|^{2} d \xi+\int_{|\xi| \leqq K}|\xi|^{-2 m}|\hat{\phi}(\xi)|^{2} d \xi \\
& \leqq K^{-2 m}\|\phi\|^{2}+\int_{|\xi| \leqq K}|\xi|^{-2 m}|\hat{\phi}(\xi)|^{2} d \xi
\end{aligned}
$$

by Parseval's relation. Now

$$
\begin{aligned}
\hat{\phi}(\xi) & =(2 \pi)^{-n / 2} \int_{R^{n}} e^{-i \xi x} \phi(x) d x \\
& =(2 \pi)^{-n / 2} \int_{|x| \leqq R}\left(\sum_{j=p}^{\infty} \frac{(-i \xi x)^{j}}{j !}\right) \phi(x) d x
\end{aligned}
$$

since $\phi$ is in $\left(I-\pi_{p}\right)\left(L_{2}\left(B_{R}^{n} ; C^{k}\right)\right)$. Therefore

$$
\begin{aligned}
|\hat{\phi}(\xi)| & \leqq(2 \pi)^{-n / 2} \sum_{j=p}^{\infty} \frac{|\xi|^{j}}{j !}\left\{\int_{|x| \leqq R}|x|^{j}|\phi(x)| d x\right\} \\
& \leqq(2 \pi)^{-n / 2} \sum_{j=n}^{\infty} \frac{|\xi|^{j}}{j !}\left\{\int_{|x| \leqq R}|x|^{2 j} d x\right\}^{1 / 2}\|\phi\| \\
& \leqq(2 \pi)^{-n / 2} \sum_{j=p}^{\infty} \frac{|\xi|^{j} R^{j+n / 2} A_{n}^{1 / 2}}{j !(2 j+n)^{1 / 2}}\|\phi\|
\end{aligned}
$$

where $A_{n}=\int_{S^{n-1}} d w$ is the area of the unit sphere in $R^{n}$. Continuing,

$$
\begin{aligned}
|\hat{\phi}(\xi)| & \leqq(2 \pi)^{-n / 2} \sum_{j=0}^{\infty} \frac{|\xi|^{j+p} R^{j+p+n / 2} A_{n}^{1 / 2}}{(j+p) !(2 j+2 p+n)^{1 / 2}}\|\phi\| \\
& \leqq(2 \pi)^{-n / 2} e^{|\xi| R} \frac{|\xi|^{p} R^{p+n / 2} A_{n}^{1 / 2}}{p !(2 p+n)^{1 / 2}}\|\phi\| .
\end{aligned}
$$


Then

$$
\begin{aligned}
\int_{|\xi| \leqq K}|\xi|^{-2 m}|\hat{\phi}(\xi)|^{2} d \xi & \leqq(2 \pi)^{-n} \frac{e^{2 K R} R^{2 p+n} A_{n}}{(p !)^{2}(2 p+n)}\left\{\int_{|\xi| \leqq K}|\xi|^{2 p-2 m} d \xi\right\}\|\phi\|^{2} \\
& \leqq(2 \pi)^{-n} \frac{e^{2 K R} K^{2 p+n-2 m} R^{2 p+n} A_{n}^{2}}{(p !)^{2}(2 p+n)(2 p+n-2 m)}\|\phi\|^{2}
\end{aligned}
$$

and so

$$
\begin{array}{rl}
\int_{R^{n}}|\xi|^{-2 m}|\hat{\phi}(\xi)|^{2} & d \xi \\
& \leqq K^{-2 m}\left[1+(2 \pi)^{-n} \frac{e^{2 K R}(K R)^{2 p+n} A_{n}^{2}}{(p !)^{2}(2 p+n)(2 p+n-2 m)}\right]\|\phi\|^{2} .
\end{array}
$$

If $K$ is taken to be $(2 p+n-2 m)^{(1-\varepsilon)} / R$, then the inequality becomes

$$
\begin{aligned}
& \int_{R^{n}}|\xi|^{-2 m}|\hat{\phi}(\xi)|^{2} d \xi \\
& \leqq \frac{R^{2 m}}{(2 p+n-2 m)^{2 m(1-\varepsilon)}} \\
& \times\left[1+(2 \pi)^{-n} \frac{e^{2(2 p+n-2 m)^{(1-\varepsilon)}}(2 p+n-2 m)^{(2 p+n)(1-\varepsilon)-1} A_{n}^{2}}{(p !)^{2}(2 p+n)}\right]\|\phi\|^{2} .
\end{aligned}
$$

To prove the lemma, it will suffice to show that the function

$$
F(p)=\frac{e^{2(2 p+n-2 m)^{(1-\varepsilon)}}(2 p+n-2 m)^{(2 p+n)(1-\varepsilon)-1}}{(p !)^{2}(2 p+n)}
$$

is bounded from above for all nonnegative integers $p$ greater than $(m-n / 2)$ by a function $G(p)$ which becomes monotone decreasing after a finite, determinable number of integers $p$. Indeed, if such a function $G(p)$ exists, then the desired constant $C_{n, m, \varepsilon}$ may be taken to be the calculable quantity

Define

$$
C_{n, m, \varepsilon}=\left[1+(2 \pi)^{-n} A_{n \geq 0 ; p>(m-n / 2)}^{2} \sup _{p(p)] .}\right.
$$

$$
G(x)=\frac{e^{2(2 x+n-2 m)^{(1-\varepsilon)}}(2 x+n-2 m)^{(2 x+n)(1-\varepsilon)-1}}{\left\{\exp \left(2 \int_{0}^{x} \log s d s\right)\right\}(2 x+n)}
$$

for all nonnegative $x$ greater than $(m-n / 2)$. Since

$$
\exp \left(2 \int_{0}^{p} \log s d s\right) \leqq(p !)^{2}
$$


for all nonnegative integers $p$, it is immediate that $F(p) \leqq G(p)$ for all nonnegative integers $p$ greater than $(m-n / 2)$. Furthermore, $G$ is monotone decreasing for large values of its argument, because a number $x_{0}$ may be easily found beyond which

$$
\begin{aligned}
\frac{d}{d x}[\log G(x)]= & \frac{4(1-\varepsilon)}{(2 x+n-2 m)^{\varepsilon}}+2(1-\varepsilon) \log (2 x+n-2 m) \\
& +\frac{2[(2 x+n)(1-\varepsilon)-1]}{(2 x+n-2 m)}-2 \log x-\frac{2}{2 x+n}
\end{aligned}
$$

is negative. This completes the proof of the lemma.

PROOF OF THE THEOREM. Let $p$ be a nonnegative integer greater than $(m-n / 2)$ and let $u$ be an element of $H_{m}\left(\boldsymbol{R}^{n} ; \boldsymbol{C}^{k}\right)$. Note that

$$
\begin{aligned}
\int_{|x| \leqq R}\left|\left(I-\pi_{p}\right) u(x)\right|^{2} d x & \sup _{\phi \in\left(I-\pi_{p}\right)\left(L_{2}\left(B_{R}^{n} ; c^{k}\right)\right) ;\|\phi\| \leqq 1}\left|\left(\phi,\left(I-\pi_{p}\right) u\right)\right|^{2} \\
& =\sup _{\phi \in\left(I-\pi_{p}\right)\left(L_{2}\left(B_{R}^{n}: c^{k}\right)\right) ;\|\phi\| \leqq 1}|(\phi, u)|^{2} \\
& =\left.\left.\sup _{\phi \in\left(I-\pi_{p}\right)\left(L_{2}\left(B_{R}^{n} ; c^{k}\right)\right) ;\|\phi\| \leqq 1}\left|\int_{R^{n}}\right| \xi\right|^{-m} \hat{\phi}(\xi) \overline{|\xi|^{m} \hat{u}(\xi)} d \xi\right|^{2} \\
& \leqq \sup _{\phi \in\left(I-\pi_{p}\right)\left(L_{2}\left(B_{R}^{n} ; C^{k}\right)\right) ;\|\phi\| \leqq 1}\left\{\int_{R^{n}}|\xi|^{-2 m}|\hat{\phi}(\xi)|^{2} d \xi\right\}\left\|\partial_{x}^{m} u\right\|^{2} \\
& \leqq \frac{C_{n, m, \varepsilon} R^{2 m}}{(2 p+n-2 m)^{2 m(1-\varepsilon)}}\left\|\partial_{x}^{m} u\right\|^{2}
\end{aligned}
$$

where $C_{n, m, \varepsilon}$ is the constant in the estimate of the lemma. The theorem then follows immediately from the observation that

$$
\int_{|x| \leqq R}|u(x)|^{2} d x=\left\|\pi_{p} u\right\|^{2}+\int_{|x| \leqq R}\left|\left(I-\pi_{p}\right) u(x)\right|^{2} d x
$$

and the proof is complete.

3. An application. After a brief sketch of the Fredholm theory of linear elliptic first-order partial differential operators in $\boldsymbol{R}^{n}$ whose first-order coefficients become constant and whose zero-order coefficients vanish outside a bounded subset of $\boldsymbol{R}^{n}$, the theorem of the preceding section will be applied toward establishing new bounds on the indices of such operators when the number of independent variables is two. (A linear first-order 
partial differential operator

$$
A u(x)=\sum_{i=1}^{n} A_{i}(x) \frac{\partial}{\partial x_{i}} u(x)+B(x) u(x)
$$

with domain $H_{1}\left(\boldsymbol{R}^{n} ; \boldsymbol{C}^{k}\right)$ is said to be elliptic if $\operatorname{det}\left|\sum_{i=1}^{n} A_{i}(x) \xi_{i}\right| \neq 0$ for all $x$ in $\boldsymbol{R}^{n}$ and all nonzero $\xi$ in $\boldsymbol{R}^{n}$.) Similar applications of the theorem in its full generality will be made in the sequel to [4]. For details relevant to the following discussion, the reader is referred to [3] and [4].

Given a positive number $R$ and a linear elliptic first-order partial differential operator $A_{0}$ with domain $H_{1}\left(\boldsymbol{R}^{n} ; C^{k}\right)$ having constant coefficients and no zero-order term, denote by $E\left(A_{0}, R\right)$ the set of all linear elliptic first-order partial differential operators with continuous zero-order coefficients and continuously differentiable first-order coefficients whose coefficients are equal to those of $A_{0}$ outside the ball $B_{R}^{n}$. Denote by $M\left(A_{0}, R\right)$ the subspace of $H_{1}\left(\boldsymbol{R}^{n} ; \boldsymbol{C}^{k}\right)$ consisting of all elements $u$ of $H_{1}\left(\boldsymbol{R}^{n} ; \boldsymbol{C}^{k}\right)$ for which $A_{0} u$ has support in $B_{R}^{n}$, and let $A$ be an operator in $E\left(A_{0}, R\right)$. It is apparent that the null space $N(A)$ of $A$ is contained in $M\left(A_{0}, R\right)$. Since the support of $A u$ is contained in $B_{R}^{n}$ for each $u$ in $M\left(A_{0}, R\right)$, the restriction of $A$ to $M\left(A_{0}, R\right)$ may be thought of as a bounded operator from $M\left(A_{0}, R\right)$ to $L_{2}\left(B_{R}^{n}, C^{k}\right)$. It is shown in [3] that the $\operatorname{dimension} \operatorname{dim} N(A)$ of $N(A)$ is finite, and, since the adjoint operator $A^{*}$ of $A$ is an operator in $E\left(A_{0}^{*}, R\right)$, it follows that $\operatorname{dim} N\left(A^{*}\right)$ is also finite. It is shown in [4] that the restriction of $A$ to $M\left(A_{0}, R\right)$ is a bounded Fredholm operator from $M\left(A_{0}, R\right)$ to $L_{2}\left(B_{R}^{n} ; C^{k}\right)$. If the number of independent variables is at least three, then the index of this Fredholm restriction of $A$ is $[\operatorname{dim} N(A)-$ $\operatorname{dim} N\left(A^{*}\right)$ ]. If the number of independent variables is two, however, the index cannot be prescribed so elegantly. In [5], it is shown that if the number of independent variables is two, then the index of the Fredholm restriction of $A$ is at least $\left[\operatorname{dim} N\left(A_{f}\right)-\operatorname{dim} N\left(A_{f}^{*}\right)-k\right]$ and at most $\min \left\{\left[\operatorname{dim} N(A)-\operatorname{dim} N\left(A^{*}\right)\right],\left[\operatorname{dim} N\left(A_{f}\right)-\operatorname{dim} N\left(A_{f}^{*}\right)\right]\right\}$, where $A_{f}$ denotes the operator in $E\left(A_{0}, R\right)$ consisting of the first-order terms of $A$. It is a corollary to the following lemma, whose proof employs the theorem of the preceding section, that the index is at least [dim $N(A)-\operatorname{dim} N\left(A^{*}\right)-2 k$ ] and at most $\left[\operatorname{dim} N(A)-\operatorname{dim} N\left(A^{*}\right)\right]$; thus the index is bounded by quantities defined by the operator $A$ alone.

LEMMA. If $A$ is an operator in $E\left(A_{0}, R\right)$ and if the number $n$ of independent variables is equal to two, then the dimension of the orthogonal complement of $A\left(M\left(A_{0}, R\right)\right)$ in $L_{2}\left(B_{R}^{2} ; C^{k}\right)$ is at least $\operatorname{dim} N\left(A^{*}\right)$ and at most $\left[\operatorname{dim} N\left(A^{*}\right)+2 k\right]$.

Proof. First note that the restriction of any element of $N\left(A^{*}\right)$ to $B_{R}^{2}$ is orthogonal in $L_{2}\left(B_{R}^{2} ; C^{k}\right)$ to $A\left(M\left(A_{0}, R\right)\right)$. Hence the dimension of the 
orthogonal complement of $A\left(M\left(A_{0}, R\right)\right)$ in $L_{2}\left(B_{R}^{2} ; C^{k}\right)$ is at least $\operatorname{dim} N\left(A^{*}\right)$.

To show that the dimension of the orthogonal complement of $A\left(M\left(A_{0}, R\right)\right)$ in $L_{2}\left(B_{R}^{2} ; C^{k}\right)$ is at most $\left[\operatorname{dim} N\left(A^{*}\right)+2 k\right]$, first suppose that an arbitrary element $v$ of $L_{2}\left(B_{R}^{2} ; C^{k}\right)$ is given. Since $A$ has bounded coefficients, there exist positive constants $c_{1}$ and $c_{2}$ for which the estimate

$$
\begin{aligned}
|(A u, v)| & \leqq\left\{\int_{|x| \leqq R}|A u(x)|^{2} d x\right\}^{1 / 2}\|v\| \\
& \leqq\left\{c_{1} \int_{|x| \leqq R}|u(x)|^{2} d x+c_{2}\left\|\partial_{x}^{1} u\right\|^{2}\right\}^{1 / 2}\|v\|
\end{aligned}
$$

holds for all $u$ in $H_{1}\left(\boldsymbol{R}^{2} ; \boldsymbol{C}^{k}\right)$. Noting the elliptic estimate $\left\|\partial_{x}^{1} u\right\| \leqq$ const $\left\|A_{0} u\right\|$ for elements $u$ of $H_{1}\left(R^{2} ; C^{k}\right)$, one then sees as a particular consequence of the theorem of the preceding section that there exists a positive constant $c$ for which the estimate

$$
|(A u, v)| \leqq c\left\{\left\|\pi_{1} u\right\|^{2}+\left\|A_{0} u\right\|^{2}\right\}^{1 / 2}\|v\|
$$

holds for all $u$ in $H_{1}\left(R^{2} ; C^{k}\right)$. Denote by $L\left(A_{0}, R\right)$ the set of all elements $u$ of $H_{1}\left(R^{2} ; C^{k}\right)$ for which $A_{0} u$ is constant in $B_{R}^{2}$. The set of ordered pairs $\left\{\left[\pi_{1} u, A_{0} u\right]: u \in L\left(A_{0}, R\right)\right\}$ is a pre-Hilbert space with respect to the norm $\left\|\left[\pi_{1} u, A_{0} u\right]\right\|=\left\{\left\|\pi_{1} u\right\|^{2}+\left\|A_{0} u\right\|^{2}\right\}^{1 / 2}$, and it follows from the above estimate that the assignment $\left[\pi_{1} u, A_{0} u\right] \rightarrow(A u, v)$ is a bounded linear functional on this pre-Hilbert space. The Riesz representation theorem then implies that there is a unique element of the completion of this pre-Hilbert space which represents this bounded linear functional. That is to say, there exists a unique $w$ in $\pi_{1}\left(L_{2}\left(R^{2} ; C^{k}\right)\right)$ and a unique $z$ in the closure of $A_{0}\left(L\left(A_{0}, R\right)\right)$ in $L_{2}\left(R^{2} ; C^{k}\right)$ for which the relation $(A u, v)=\left(\pi_{1} u, w\right)+\left(A_{0} u, z\right)$ holds for all $u$ in $L\left(A_{1}, R\right)$. Note that both $w$ and $z$ are constant in $B_{R}^{2}$ and that $w$ vanishes in $R^{2}-B_{R}^{2}$.

Now let arbitrary elements $v_{1}, \cdots, v_{2 k+1}$ of the orthogonal complement of $A\left(M\left(A_{0}, R\right)\right)$ in $L_{2}\left(B_{R}^{2} ; C^{k}\right)$ be given. The lemma will be proved if it can be shown that there exist scalars $\alpha_{1}, \cdots, \alpha_{2 k+1}$, not all zero, such that the linear combination $\alpha_{1} v_{1}+\cdots+\alpha_{2 k+1} v_{2 k+1}$ can be extended to an element of $N\left(A^{*}\right)$. It follows from the above discussion that there exist elements $w_{1}, \cdots, w_{2 k+1}$ of $\pi_{1}\left(L_{2}\left(\boldsymbol{R}^{2} ; \boldsymbol{C}^{k}\right)\right)$ and elements $z_{1}, \cdots, z_{2 k+1}$ of the closure of $A_{0}\left(L\left(A_{0}, R\right)\right)$ in $L_{2}\left(R^{2} ; C^{k}\right)$ for which the relation

$$
\left(A u, v_{j}\right)=\left(\pi_{1} u, w_{j}\right)+\left(A_{0} u, z_{j}\right)
$$

holds for all $u$ in $L\left(A_{0}, R\right)$ and for each $j, 1 \leqq j \leqq 2 k+1$. Since the elements $w_{1}, \cdots, w_{2 k+1}^{\prime}$ and $z_{1}, \cdots, z_{2 k+1}$ are constant $\boldsymbol{C}^{k}$-valued functions in $B_{R}^{2}$, there are scalars $\alpha_{1}, \cdots, \alpha_{2 k+1}$, not all zero, which are such that the functions $\sum_{j=1}^{2 k+1} \alpha_{j} w_{j}$ and $\sum_{j=1}^{2 k+1} \alpha_{j} z_{j}$ vanish inside $B_{R}^{2}$. (In particular, the 
function $\sum_{j=1}^{2 k+1} \alpha_{j} w_{j}$ vanishes on all of $\boldsymbol{R}^{n}$.) Then the relation

$$
\left(A u, \sum_{j=1}^{2 k+1} \alpha_{j} v_{j}\right)=\left(A_{0} u, \sum_{j=1}^{2 k+1} \alpha_{j} z_{j}\right)
$$

holds for all $u$ in $L\left(A_{0}, R\right)$. Now suppose that $u$ is an arbitrary element of $H_{1}\left(R^{2}, C^{k}\right)$. It is shown in [4] that $u$ may be uniquely written as a sum $u=u_{1}+u_{2}$, where $u_{1}$ and $u_{2}$ are in $L\left(A_{0}, R\right)$ and $M\left(A_{0}, R\right)$, respectively. Then one has

$$
\begin{aligned}
\left(A u, \sum_{j=1}^{2 k+1} \alpha_{j} v_{j}-\sum_{j=1}^{2 k+1} \alpha_{j} z_{j}\right)= & \left(A u_{1}, \sum_{j=1}^{2 k+1} \alpha_{j} v_{j}\right)-\left(A u_{1}, \sum_{j=1}^{2 k+1} \alpha_{j} z_{j}\right) \\
& +\left(A u_{2}, \sum_{j=1}^{2 k+1} \alpha_{j} v_{j}\right)-\left(A u_{2}, \sum_{j=1}^{2 k+1} \alpha_{j} z_{j}\right) .
\end{aligned}
$$

The first and second terms on the right-hand side cancel, since

$$
\left(A u_{1}, \sum_{j=1}^{2 k+1} \alpha_{j} v_{j}\right)=\left(A_{0} u_{1}, \sum_{j=1}^{2 k+1} \alpha_{j} z_{j}\right)
$$

and since the coefficients of $A$ are equal to the coefficients of $A_{0}$ in $R^{2}-B_{R}^{2}$. The third term on the right-hand side is zero, since $u_{2}$ is in $M\left(A_{0}, R\right)$ and since each $v_{j}$ is orthogonal to $A\left(M\left(A_{0}, R\right)\right)$. The fourth term on the righthand side is zero, since the support of $A u_{2}$ is contained in $B_{R}^{2}$. Consequently,

$$
\left(A u, \sum_{j=1}^{2 k+1} \alpha_{j} v_{j}-\sum_{j=1}^{2 k+1} \alpha_{j} z_{j}\right)=0
$$

for all $u$ in $H_{1}\left(R^{2} ; C^{k}\right)$. This is to say that $\left(\sum_{j=1}^{2 k+1} \alpha_{j} v_{j}-\sum_{j=1}^{2 k+1} \alpha_{j} z_{j}\right)$ is an extension of $\sum_{j=1}^{2 k+1} \alpha_{j} v_{j}$ to an element of $N\left(A^{*}\right)$, and the lemma is proved.

\section{REFERENCES}

1. A. Friedman, Partial differential equations of parabolic type, Prentice-Hall, Englewood Cliffs, N.J., 1964. MR 31 \#6062.

2. P. D. Lax and R. S. Phillips, Scattering theory, Pure and Appl. Math., vol. 26, Academic Press, New York, 1967. MR 36 \#530.

3. H. F. Walker, On the null-spaces of first-order elliptic partial differential operators in $R^{n}$, Proc. Amer. Math. Soc. 30 (1971), 278-286. MR 43 \#6583.

4. - A Fredholm theory for a class of first-order elliptic partial differential operators in $R^{n}$, Trans. Amer. Math. Soc. 165 (1972), 75-86.

Department of Mathematics, TeXas Tech University, Lubbock, TeXas 70409 